\title{
Enhanced Survival of Spiral Ganglion Cells After Cessation of Treatment with Brain-Derived Neurotrophic Factor in Deafened Guinea Pigs
}

\author{
Martijn J. H. AgterberG ${ }^{1}$, Huib Versnel ${ }^{1}$, Lotte M. van Dijk ${ }^{1}$, John C. M. J. de Groot ${ }^{1}$, \\ AND SJAAK F. L. KLIS ${ }^{1}$ \\ ${ }^{1}$ Department of Otorhinolaryngology, Rudolf Magnus Institute of Neuroscience, University Medical Center Utrecht, Room G.02.531, \\ P. O. Box 85500, 3508 GA Utrecht, The Netherlands
}

Received: 4 November 2008; Accepted: 20 March 2009; Online publication: 14 April 2009

\begin{abstract}
Exogenous delivery of neurotrophic factors into the cochlea of deafened animals rescues spiral ganglion cells (SGCs) from degeneration. To be clinically relevant for human cochlear implant candidates, the protective effect of neurotrophins should persist after cessation of treatment and the treated SGCs should remain functional. In this study, the survival and functionality of SGCs were investigated after temporary treatment with brain-derived neurotrophic factor (BDNF). Guinea pigs in the experimental group were deafened, and 2 weeks later, the right cochleae were implanted with an electrode array and drug delivery cannula. BDNF was administered to the implanted cochleae during a 4-week period via a mini-osmotic pump. After completion of the treatment, the osmotic pumps were removed. Two weeks later, the animals were killed and the survival of SGCs was analyzed. To monitor the functionality of the auditory nerve, electrically evoked auditory brainstem responses (eABRs) were recorded in awake animals throughout the experiment. BDNF treatment resulted in enhanced survival of SGCs 2 weeks after cessation of the treatment and prevented the decreases in size and circularity that are seen in the untreated contralateral cochleae. The amplitude of the suprathreshold eABR
\end{abstract}

Correspondence to: Sjaak F. L. Klis · Department of Otorhinolaryngology, Rudolf Magnus Institute of Neuroscience · University Medical Center Utrecht - Room G.02.531, P. O. Box 85500, 3508 GA Utrecht, The Netherlands. Telephone: +31-88-7557724; fax: +31-30-2541922; email: s.klis@umcutrecht.nl response in BDNF-treated animals was significantly larger than in deafened control animals and comparable to that in normal-hearing control animals. The amplitude in the BDNF-treated group did not decrease significantly after cessation of treatment. The eABR latency in BDNF-treated animals was longer than normal and comparable to that in deafened control animals. These morphological and functional findings demonstrate that neurotrophic intervention had a lasting effect, which is promising for future clinical application of neurotrophic factors in implanted human cochleae.

Keywords: auditory nerve, cochlear implant, deafness, degeneration, electrically evoked auditory brainstem response, neurotrophic factors

\section{INTRODUCTION}

A progressive loss of spiral ganglion cells (SGCs) after loss of inner hair cells is presented in several animal models (Spoendlin 1975; Webster and Webster 1981). In these models, electrical stimulation (Lousteau 1987; Hartshorn et al. 1991; Leake et al. 1991) and administration of neurotrophins (Staecker et al. 1996; Miller et al. 1997; Gillespie and Shepherd 2005; Pettingill et al. 2007) via a cochlear implant are effective in preventing SGCs from degeneration after induced deafness. The preservation of SGCs might be important since the success of the cochlear implant is thought to be related to the number of excitable SGCs. In the majority of studies, neurotrophic treatment was started within the 
first 2 weeks after deafening (see review, Gillespie and Shepherd 2005). It has been shown that neurotrophic treatment is also effective after degeneration has set in, a condition that mimics the clinical situation (Gillespie et al. 2004; Yamagata et al. 2004; Wise et al. 2005; Miller et al. 2007; Agterberg et al. 2008; Glueckert et al. 2008) . In these studies, intracochlear infusion of neurotrophins by means of an osmotic pump system was started 2-6 weeks after deafening. Neurotrophic treatment prevented SGC degeneration, but SGCs treated with BDNF were larger than SGCs in normal cochleae and their myelin layers were reduced (Richardson et al. 2005; Shepherd et al. 2005, 2008; Agterberg et al. 2008; Glueckert et al. 2008). These morphological changes might reflect a suboptimal condition. Nevertheless, SGCs treated with neurotrophins remained electrically excitable and animals treated with neurotrophins showed reduced thresholds of electrically evoked auditory brainstem responses (eABRs) as compared to animals in deafened control groups infused with artificial perilymph (Shinohara et al. 2002; Yamagata et al. 2004; Shepherd et al. 2005; Maruyama et al. 2008).

An important question with respect to clinical application of neurotrophins is whether continuous treatment is necessary for lasting protective effects. According to the neurotrophin hypothesis and based upon findings in other sensory systems (Montero and Hefti 1988; Mansour-Robaey et al. 1994), it is predicted that cessation of neurotrophic treatment would result in degeneration of SGCs. This prediction has been addressed in recent studies, which yielded contradictory results. Gillespie et al. (2003) and Shepherd et al. (2008) reported an abnormally rapid degeneration of SGCs after cessation of BDNF treatment. Two weeks after cessation, the number of SGCs was not significantly different from that in deafened, untreated cochleae. In addition, Shepherd et al. (2008) found that chronic electrical stimulation partially reduced the rate of SGC loss in the basal cochlear turn, i.e., the area adjacent to the electrode array. In contrast to these findings with BDNF, Maruyama et al. (2008) demonstrated that SGC survival and electrical responsiveness were well preserved after cessation of intracochlear infusion with glial cell line-derived neurotrophic factor (GDNF).

In the present study, we investigated the effects of cessation of BDNF treatment. Treatment was started 2 weeks after deafening, when degeneration had set in. Similar to Gillespie et al. (2003), we used BDNF as neurotrophic factor, and similar to Maruyama et al. (2008), we recorded eABRs to monitor the excitability of the SGCs. Light microscopy was used to determine the SGC packing density and to examine morphological features, such as cell size and shape (circularity). The functionality of the SGCs was assessed with the suprathreshold amplitude (Hall 1990) and latency of the first negative eABR peak.

\section{METHODS}

\section{Animals and experimental design}

Twenty albino female guinea pigs (strain: Dunkin Hartley; weighing 250-350 g) were purchased from Harlan Laboratories (Horst, The Netherlands) and housed in the animal care facility of Utrecht University. All animals had free access to both food and water and were kept under standard laboratory conditions. Lights were on between 7:00 AM and 7:00 PM. Temperature $\left(21^{\circ} \mathrm{C}\right)$ and humidity $(60 \%)$ were kept constant.

Six guinea pigs were bilaterally deafened and 2 weeks thereafter implanted in the right cochlea with an electrode array and cannula. Consecutively, they received BDNF during a period of 4 weeks. After completion of BDNF treatment, the osmotic pumps were removed. Two weeks after BDNF treatment (i.e., 8 weeks after deafening), these animals were killed and processed for histology. For histological analysis, a comparison was made between BNDF-treated and untreated ears in the same animals; furthermore, the histological data of BDNF-treated ears were compared to data from normal cochleae ( $n=4$ animals) presented in a previous paper (Agterberg et al. 2008).

After implantation, eABRs in BDNF-treated animals were recorded weekly to assess auditory function in relation to electrical stimulation of the treated cochleae. This paradigm is schematically explained in Figure $1 \mathrm{~A}$. The eABR data of the treated animals were compared to eABRs in both normal-hearing and deafened untreated animals. For this, we used three groups of control animals (Fig. 1 B-D). A group of two animals were deafened and implanted in the right cochlea with a regular electrode array (without drug delivery cannula) 2 weeks thereafter, and eABRs were recorded up to more than 8 weeks after deafening (Fig. 1 B). A second group of eight normal-hearing control animals were implanted in the right cochlea with a regular electrode array and eABRs were recorded weekly (Fig. $1 \mathrm{C}$ ). In order to examine the effect of deafening on the eABRs in the same animals, these animals were deafened after 4 weeks and killed another 6 weeks later (in one of these animals, eABRs could not be recorded anymore in later sessions due to electrode failure). A third group of four animals were implanted as the second group but killed after 4 weeks while in normalhearing condition (Fig. $1 \mathrm{D}$ ). All surgical and experimental procedures were approved by the Animal Care and Use Committee of Utrecht University (DEC-UMC \# 03.04.036).

\section{Deafening procedure}

Animals were anesthetized with Domitor ${ }^{\circledR}$ (medetomidine hydrochloride; $10 \mathrm{mg} / \mathrm{kg}$, i.m.) and Ketanest-S ${ }^{\circledR}$ ((S)-ketamine; $40 \mathrm{mg} / \mathrm{kg}$, i.m.). Before the deafening 


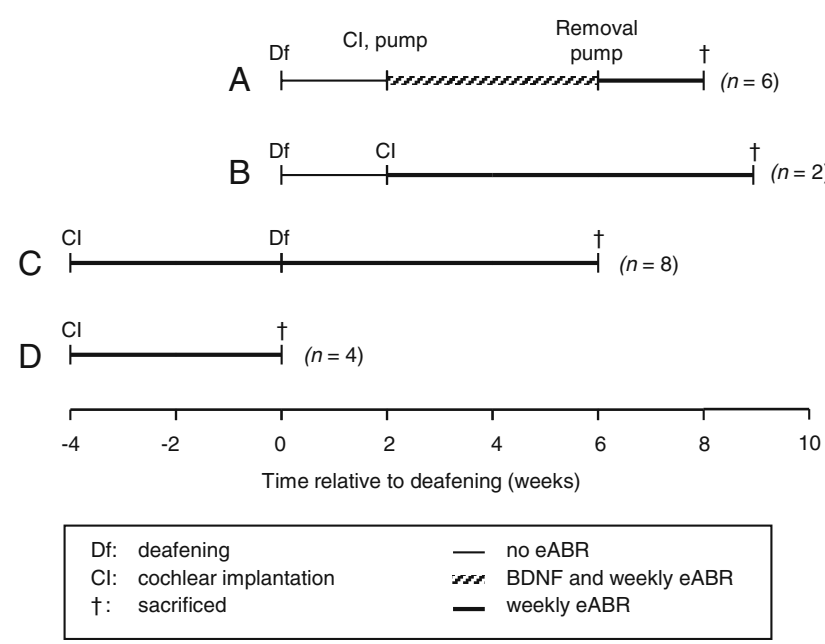

FIG. 1. Treatment schedule of four different animal cohorts (A-D). A Deafened and 2 weeks later implanted and treated with BDNF; B deafened and 2 weeks later implanted; $\mathbf{C}$ first implanted and 4 weeks later deafened; D only implanted. Deafening was performed systemically affecting both ears. Cochlear implantation (A-D) and BDNF treatment (A) was applied to the right ear. After implantation, eABRs were regularly recorded in each group (by electrically stimulating the implanted right ear). For electrophysiological analysis, eABRs of the BDNF-treated animals (A) were compared to eABRs of normal-hearing animals (C before deafening, $\mathbf{D}$ ) and to eABRs of deafened animals (B, C after deafening). Note that data in normalhearing and deafened conditions were obtained in the same animals (C). For histological analysis, the main comparison was made within the animals treated with BDNF (A): BNDF-treated right ears were compared to untreated left ears.

procedure, acoustically evoked ABRs (aABRs) were recorded to check hearing thresholds. Animals were injected subcutaneously with kanamycin $(400 \mathrm{mg} / \mathrm{kg}$, s.c.) followed (15-60 min later) by slow intravenous infusion of furosemide $(100 \mathrm{mg} / \mathrm{kg}$, i.v.). This procedure, originally reported by West et al. (1973), has been shown to eliminate almost all cochlear hair cells (Gillespie et al. 2003; Versnel et al. 2007). For the intravenous infusion of furosemide, the right jugular vein was exposed and a catheter was inserted. A successful insertion was confirmed with withdrawal of blood into the catheter.

\section{Implantation and cochlear infusion}

Animals were anesthetized with Domitor ${ }^{\circledR}(10 \mathrm{mg} / \mathrm{kg}$, i.m.) and Ketanest- $S^{\circledR}(40 \mathrm{mg} / \mathrm{kg}$, i.m.). In the control animals the right cochleae were implanted with an eight-electrode array (Cochlear ${ }^{\circledR}$, platinum ring electrodes of $0.3 \mathrm{~mm}$ width, inter-electrode distance $0.75 \mathrm{~mm}$ ). In the experimental animals, the right cochleae were implanted with a six-electrode array (same electrode configuration as eight-electrode array) with drug delivery cannula $(\sim 80 \mathrm{~mm}, \mathrm{ID}=0.8 \mathrm{~mm})$ with a tip of $\sim 20 \mathrm{~mm}$ (ID=0.12 mm; OD=0.16 mm) designed for guinea pigs $\left(\operatorname{Cochlear}^{\circledR}\right)$, resembling the array-cannula device described by Shepherd and Xu (2002). The right bulla was exposed retro-auricularly and a small hole was drilled to visualize the cochlea. The array was inserted 3-4 $\mathrm{mm}$ through a cochleostomy in the basal turn near the round window. The array cable was fixed onto the bulla with dental cement (Ketac-Cem Aplicap, ESPE dental supplies, Utrecht, The Netherlands) and connected to the skull with a screw (Brown et al. 1993) and dental cement. An Alzet ${ }^{\circledR}$ mini-osmotic pump (model 2004; flow rate $0.25 \mu \mathrm{l} / \mathrm{h}$; reservoir $200 \mu \mathrm{l}$ ) was attached to the cannula and inserted into a subcutaneous pocket. The incision was closed in two layers with Vicryl $^{\circledR}$. The cannula and the mini-osmotic pump were filled with BDNF (PeproTech Inc., Rocky Hill, NJ, USA) solution $(100 \mu \mathrm{g} / \mathrm{ml})$. This concentration was chosen because concentrations of neurotrophins in this range $(50-100 \mu \mathrm{g} / \mathrm{ml})$ have proved to be effective in several studies (Gillespie et al. 2003, 2004; Yamagata et al. 2004; Wise et al. 2005; Miller et al. 2007; Agterberg et al. 2008; Shepherd et al. 2008). Bovine serum albumin (1\%) was added to the BDNF solution. The pumps were incubated in sterile saline for $48 \mathrm{~h}$ at $37^{\circ} \mathrm{C}$ to guarantee a constant flow rate at implantation.

\section{Auditory brainstem responses}

aABRs and eABRs were measured once or twice a week in awake and freely moving animals. All electrophysiological recordings were performed in a sound-attenuated chamber. The aABRs and eABRs were recorded with three stainless steel screws $(8.0 \times$ $1.2 \mathrm{~mm}$ ) inserted into the skull bone $1 \mathrm{~cm}$ posterior to bregma, $2 \mathrm{~cm}$ anterior to bregma, and $1 \mathrm{~cm}$ lateral from bregma, respectively (Mitchell et al. 1997). Stimulus generation and signal acquisition were controlled with custom-written software and a personal computer. The stimuli were synthesized and attenuated using a Tucker-Davis Technologies TDT3 system (modules RP2, PA5 (2x) and SA1). The responses were amplified differentially using a Princeton Applied Research 113 pre-amplifier (amplification 5,000; band pass filter $0.1-10 \mathrm{kHz}$ ) with the posterior and anterior screws as active and reference electrodes, respectively, and the lateral screw as ground electrode. The amplified signal was digitized by the TDT3 system (module RP2) and made available for off-line analysis.

\section{Acoustically evoked auditory brainstem responses}

Broadband click stimuli consisting of monophasic rectangular pulses (width $20 \mu \mathrm{s}$; interstimulus interval $99 \mathrm{~ms}$ ) were presented in free field, using a Blaupunkt speaker (PCxb352; $4 \Omega ; 30 \mathrm{~W}$ ) positioned $10 \mathrm{~cm}$ above the awake guinea pig. Threshold was defined as the sound level at which the aABR was just visible. The click stimuli were presented from $75 \mathrm{~dB}$ above the average 
threshold of normal-hearing animals ( $110 \mathrm{~dB}$ peSPL) down to threshold in 10-dB steps. Animals with a threshold shift of $>50 \mathrm{~dB}$, measured 14 days after deafening, were included as "deafened".

\section{Electrically evoked auditory brainstem responses}

After cochlear implantation, eABRs were recorded. Monophasic rectangular pulses generated and attenuated by the TDT3 system were converted to current pulses by a linear stimulus isolator (type A395, World Precision Instruments). The current pulses (width $20 \mu \mathrm{s}$; interstimulus interval $99 \mathrm{~ms}$ ) were presented with alternating polarity to the most apical intracochlear electrode using the lateral screw in the skull as return electrode (monopolar eABR recordings). To define the peaks in the eABRs, they were compared to the peaks in the aABRs (see Fig. 2). The first positive peak $\left(\mathrm{P}_{1}\right)$ in the eABR recordings was often obscured
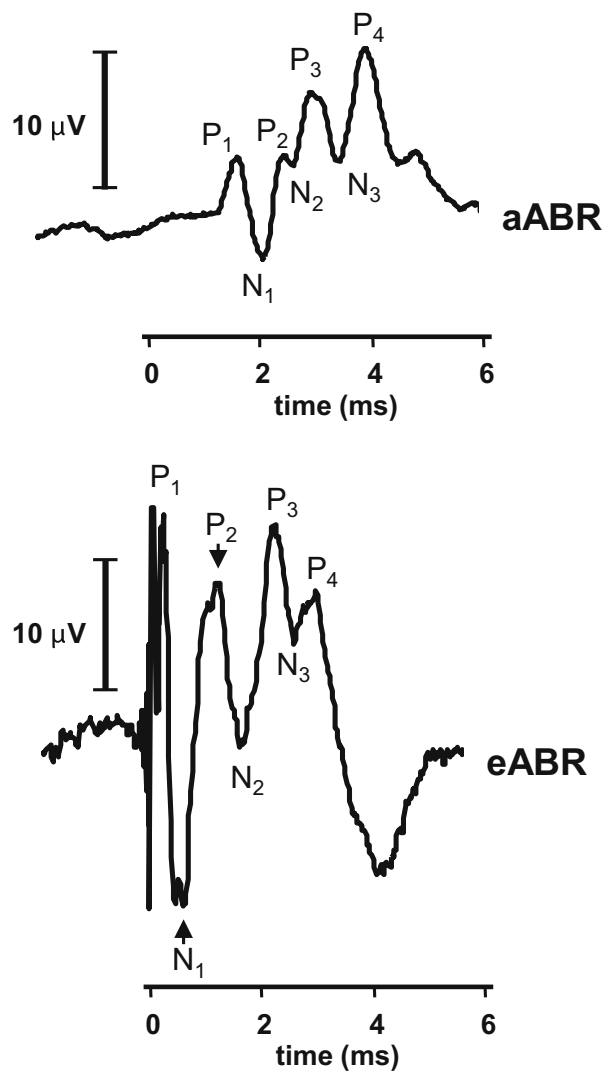

FIG. 2. A typical example of an aABR (top) evoked in a normalhearing animal, with clicks of $45 \mathrm{~dB} \mathrm{nSL}$ and an eABR (bottom) evoked with $400-\mu \mathrm{A}$ pulses. Stimulus onset was at time 0 . Note the large stimulus artifact in the eABR before peak $P_{1}$. The peak-to-peak amplitude, $\mathrm{N}_{1}-\mathrm{P}_{2}$, of the eABR was measured to assess the functional status of the auditory nerve. When the $\mathrm{N}_{1}$ and/or $\mathrm{P}_{2}$ consisted of more than one subpeak, the first subpeak on each site of the fast rising part of the eABR complex (indicated with the arrows) is used in the analysis. by the electrical artifact. In approximately half of the animals, in at least some of the recordings, the third positive peak $\left(\mathrm{P}_{3}\right)$ was influenced by the digastric muscle response (Hall 1990). The first negative peak $\left(\mathrm{N}_{1}\right)$ and second positive peak $\left(\mathrm{P}_{2}\right)$ were always clearly visible and not obscured by the electrical artifact or by the digastric muscle response. Therefore, wave $\mathrm{N}_{1}-\mathrm{P}_{2}$ was analyzed. The $\mathrm{N}_{1}-\mathrm{P}_{2}$ amplitude, $\mathrm{N}_{1}-\mathrm{P}_{2}$ threshold, and $\mathrm{N}_{1}$ latency were determined. The $\mathrm{N}_{1}-\mathrm{P}_{2}$ amplitude was found to correlate well with SGG density (Hall 1990), and therefore, it was considered an appropriate parameter to examine excitability of BDNF treated SGCs. Thresholds were defined as the stimulus level that evoked a $2.0-\mu \mathrm{V}$ (determined with interpolation) reproducible waveform. Stimuli were presented from $400 \mu \mathrm{A}$ down to threshold with 2 -dB steps. Higher current levels would evoke a whisker response. The $\mathrm{N}_{1}-\mathrm{P}_{2}$ amplitudes for all current levels in the experimental and control animals were analyzed using repeated-measures analysis of variance ( $r m$ ANOVA) and $t$ tests were used to analyze measurements at single suprathreshold current levels.

\section{Cessation of BDNF treatment}

After 4 weeks of BDNF treatment, the animals were anesthetized with Domitor $^{\circledR}(10 \mathrm{mg} / \mathrm{kg}, \mathrm{i} . \mathrm{m}$.$) and$ Ketanest-S ${ }^{\circledR}(40 \mathrm{mg} / \mathrm{kg}, \mathrm{i} . \mathrm{m}$.). After the connection of the cannula with the osmotic pump was checked, the cannula was cut off at about $40 \mathrm{~mm}$ from the cochlea and the osmotic pumps were removed. The open end of the remaining cannula was sutured with Vicryl ${ }^{\circledR}$. The electrode array was left in place to record eABRs after termination of the BDNF infusion. Two weeks after cessation of BDNF treatment, the animals were euthanized for histology.

\section{Histology}

Immediately after the final eABR measurements, the left and right cochleae were fixed by intralabyrinthine perfusion with a fixative consisting of $3 \%$ glutaraldehyde, $2 \%$ formaldehyde, $1 \%$ acrolein, and $2.5 \%$ DMSO in $0.08 \mathrm{M}$ sodium cacodylate buffer ( $\mathrm{pH} 7.4$ ) followed by immersion in the same fixative for $3 \mathrm{~h}$ at room temperature. Histological processing of the cochleae was carried out according to our standard protocol (De Groot et al. 1987). After decalcification with EDTA, the cochleae were immersed in $1 \% \mathrm{OsO}_{4}$ containing $1 \% \mathrm{~K}_{4} \mathrm{Ru}(\mathrm{CN})_{6}$ for $2 \mathrm{~h}$ at $4^{\circ} \mathrm{C}$ and then rinsed, dehydrated, and embedded in toto in Spurr's low-viscosity resin. After polymerization, cochleae were divided into two halves along a standardized midmodiolar plane, and these were re-embedded in fresh resin. Semithin $(1 \mu \mathrm{m})$ sections stained with 
methylene blue and azur II were used for light microscopical evaluation and quantitative analyses.

The efficacy of the deafening procedure was assessed, in addition to aABR thresholds, by counting the number of remaining inner hair cells (IHCs) and outer hair cells (OHCs) at seven different locations along the basilar membrane at a half-turn spacing (B1, B2, M1, M2, A1, A2, and A3; Fig. 3).

Determination of SGC packing densities was performed as described previously (Versnel et al. 2007; Agterberg et al. 2008). SGC packing densities were determined using digitized light microscopical images of the spiral ganglia taken from five different cochlear locations (B1, B2, M1, M2, and A1; Fig. 3). Using the image analysis program NIH Image (version 1.63; US National Institutes of Health, Bethesda, MD), the bony boundaries of Rosenthal's canal were outlined and its cross-sectional area (in $\mathrm{mm}^{2}$ ) was calculated. The number of SGC perikarya was counted at each location. The following populations were included in the counts: (1) all perikarya demonstrating the morphological determinants typical of type-I and type-II SGCs (for details, see Romand and Romand 1984); (2) partial and complete profiles of perikarya; and (3) perikaya with and without evident nucleus or nucleoli. SGC packing density was calculated by dividing the number of SGCs by the cross-sectional area of Rosenthal's canal and expressed as the mean number of SGCs per square millimeter.

The cellular features perikaryal area and cell circularity - as a measure of perikaryal cell size and cell shape, respectively-were selected for further quantitative analysis. Because there is a considerable regional variation in SGC perikaryal area within the cochlea (Leake et al. 1999), measurements were performed in digitized light microscopical images of the spiral ganglion at one specific location in the basal

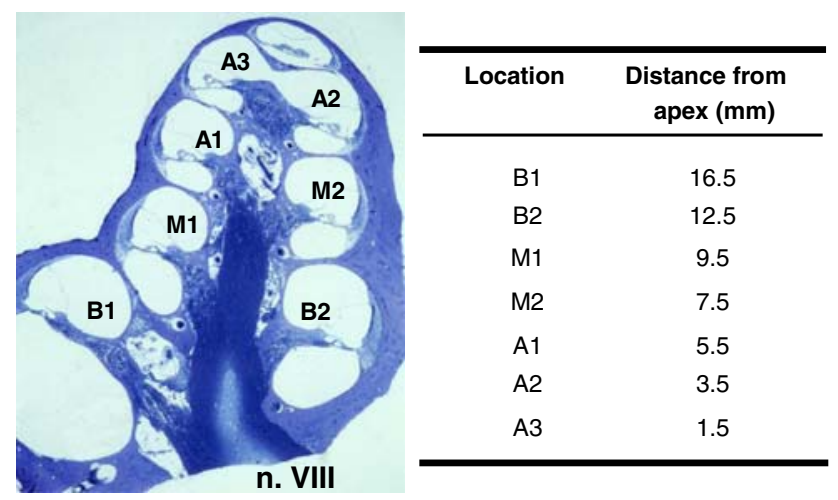

FIG. 3. Light micrograph of a midmodiolar section $(1 \mu \mathrm{m})$ of a normal guinea pig cochlea showing the different locations at which SGCs were examined. The distance from the apex of the locations B1 through A3 are specified in the table. $n$. VIII cochlear nerve. turn (location B2 in Fig. 3). This location was chosen, because (1) the effect of BDNF on SGC packing densities is most prominent in the basal turn, and (2) it was occasionally not possible to determine SGC packing densities for location $\mathrm{B}$, which is near to the hook region, due to tangential sectioning of Rosenthal's canal. Only type-I SGCs with an evident nucleus were measured. Perikaryal area was determined by outlining the myelin sheath surrounding the type-I perikaryon. Cell circularity is a feature that can be measured directly in NIH Image after delineating the cell's perimeter, i.e., the myelin sheath. It is calculated as follows: $4 \pi \times A / L^{2}$, where $A$ is the area and $L$ is the perimeter (circularity is 1 for a perfect circle and less than 1 for an imperfect circle, e.g., 0.78 for a square).

Statistical analyses were performed using SPSS $^{\circledR}$ for Windows (version 15.0.1). SGC packing densities at the different locations (B1-A1) were analyzed using repeated-measures analysis of variance (rm ANOVA). Statistical comparisons of perikaryal area and cell circularity at location $\mathrm{B} 2$ were made using paired $t$ tests.

\section{RESULTS}

\section{Effects of deafening procedure}

Two weeks after the deafening procedure, all control and experimental animals demonstrated threshold shifts of $>60 \mathrm{~dB}$ for click-evoked aABRs. This severe hearing loss was microscopically confirmed. All hair cells at cochlear locations B1-A1 were lost, except in two animals (one control animal and one experimental animal). In these animals, a few inner hair cells still remained.

\section{SGC packing densities}

Figure 4 shows light micrographs of Rosenthal's canal at cochlear location B2, providing typical examples of SGCs in cochleae of normal-hearing (A) and deafened animals (B-D), and in the left untreated and right BDNF-treated cochlea of an experimental animal (E, F). In the normal cochlea, Rosenthal's canal contained the full complement of SGCs and nerve fibers embedded in a matrix consisting of vascularized connective tissue (Fig. 4A). Two weeks after deafening, the cellular distribution within the spiral ganglia was similar to normal (Fig. 4B). Figure 4C illustrates the dramatic loss of SGCs in the left cochlea of a control animal that was examined 6 weeks after deafening. In the right cochlea, which was electrically stimulated for short periods with the purpose to record eABRs, the loss of SGCs was equally dramatic (Fig. 4D). This indicates that brief electrical stimulation did not prevent degeneration. Eight weeks after deafening, a dramatic loss of SGCs 

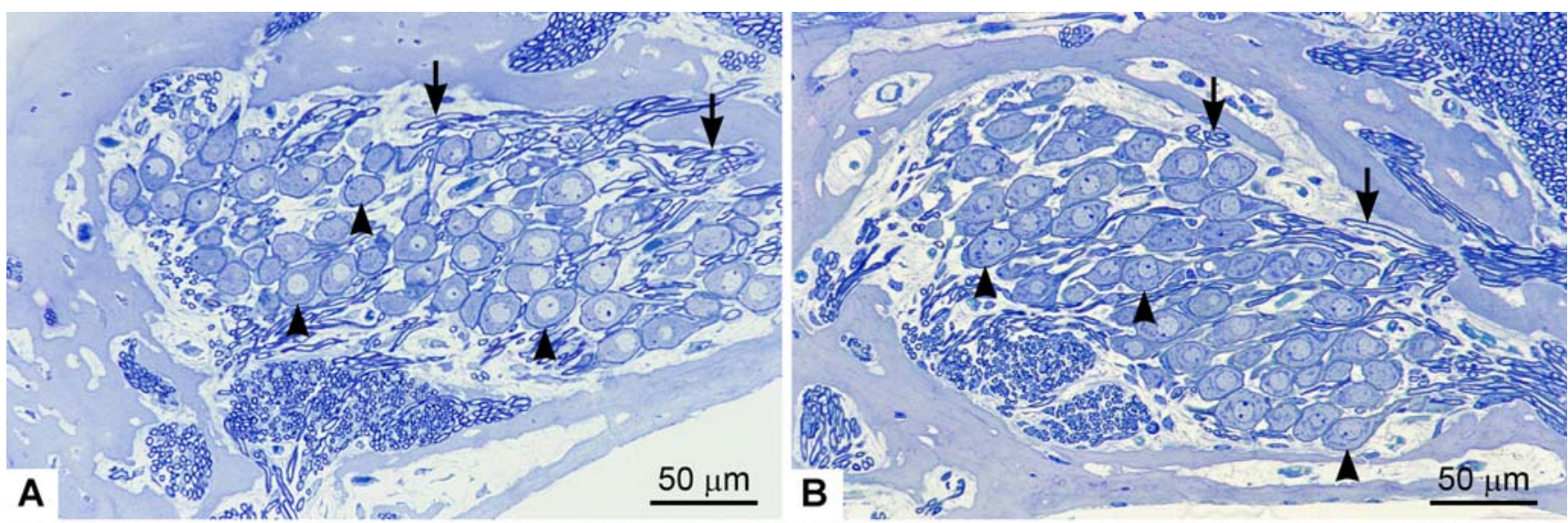

A
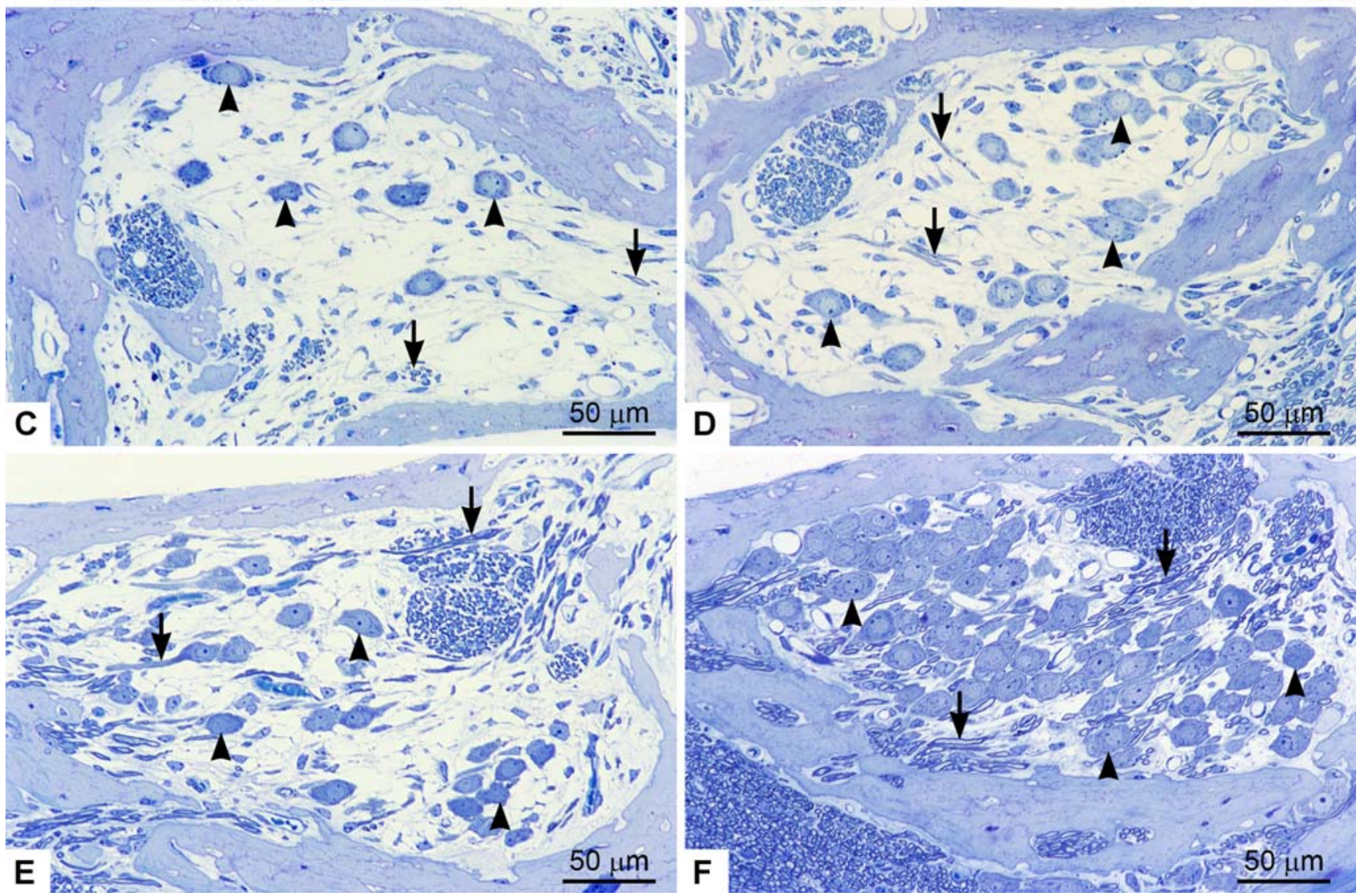

FIG. 4. Light micrographs of Rosenthal's canal at location B2 (upper basal turn) with the distribution of SGCs (arrowheads) and nerve fibers (arrows). SGCs and nerve fibers in the spiral ganglion from $\mathbf{A}$ a normal cochlea, $\mathbf{B}$ a cochlea 2 weeks after deafening, $\mathbf{C}$ a left cochlea 6 weeks after deafening, $\mathbf{D}$ the right implanted cochlea

was evident in the untreated, contralateral cochlea of a BDNF-treated animal (Fig. 4E). The surviving SGCs had lost their characteristic ovoid shape and, instead, had acquired a more elongated or dendritic appearance. In the BDNF-treated cochlea of the same animal, 2 weeks after cessation of BDNF treatment, no signs of degeneration were observed (Fig. 4F). The amount of SGCs was comparable to that in the normal cochlea, and the SGCs kept their characteristic ovoid shape. from the same animal as in $(\mathbf{C}), \mathbf{E}$ the left cochlea of an animal 8 weeks after deafening, and $\mathbf{F}$ the right implanted cochlea treated with BDNF from the same animal as in (E) (2 weeks after cessation of treatment).

Figure 5 compares SGC packing densities, at cochlear locations from B1 through A1, averaged across animals $(n=6)$, in BDNF-treated cochleae with those in the untreated contralateral cochleae and with normal data (dashed lines). The data confirm the result shown in Figure 4E, F. SGC packing densities in BDNF-treated cochleae were normal at locations B1M2 and by a factor 3 larger than those in untreated cochleae (rm ANOVA: $F(1,5)=65, p<0.001$ ). 


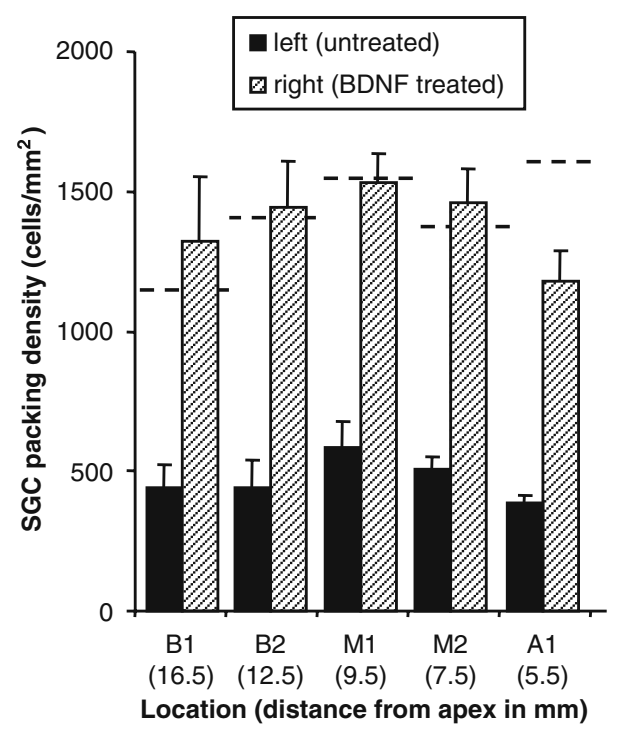

FIG. 5. Mean SGC packing densities at cochlear locations B1, B2, $M 1, M 2$, and $A 1$ in the left (untreated) and right (BDNF-treated) cochlea of deafened animals $(n=6)$. Dashed lines represent SGC densities at locations B1-A1 in normal cochleae. Error bars: SEM.

It is possible that electrical stimulation during eABR recordings contributed to the enhanced survival of SGCs (cf. Mitchell et al. 1997). Therefore, within untreated deafened animals, we compared the SGC packing densities in the stimulated implanted ears (see example in Fig. 4D) to those in the nonstimulated contralateral ears (see Fig. 4C). There were no statistically significant differences in SGC packing density between stimulated implanted ears and nonstimulated contralateral ears ( $r m$ ANOVA: $F(1,5)=3.2, p>0.1$ ).

\section{Perikaryal area and circularity}

Figure 6 shows the mean values of the perikaryal area (Fig. 6A) and circularity (Fig. 6B) of SGCs at cochlear location B2. The perikaryal area of BDNF-treated SGCs was larger than that of SGCs in the untreated contralateral cochleae with a difference of $25 \%$ (paired $t$ test, $p<0.05$ ). Circularity of BDNF-treated SGCs was larger than that of SGCs in the contralateral cochleae with a difference of $8 \%$ (paired $t$ test, $p<$ 0.05). Perikaryal area and circularity of BDNF-treated SGCs were not statistically different from perikaryal area and circularity of SGCs in normal-hearing guinea pigs. SGCs in the implanted cochleae that were electrically stimulated during eABR recordings were larger than those in the unstimulated contralateral ears (data not shown, 12\%, paired $t$ test, $p<0.05$ ). There was no significant difference in circularity of SGCs in the implanted ears as compared to the unstimulated contralateral ears (paired $t$ test, $p>0.2$ ).

\section{Electronically evoked auditory brainstem responses}

Figure 7 shows eABR recordings in two animals (top and middle row) in the normal $(\mathrm{A}, \mathrm{C})$ and deafened (B, D) condition. The decrease in $\mathrm{N}_{1}-\mathrm{P}_{2}$ amplitude after deafening was prominent in the recordings after deafening for current levels of 318 and $400 \mu \mathrm{A}$. The thresholds in these examples did not change after deafening. The $\mathrm{P}_{3}$ of the eABR recordings in $g p$-spO2 $(\mathrm{A}, \mathrm{B})$ at stimulus intensities of 400 and $318 \mu \mathrm{A}$ was influenced by the digastric muscle response (arrow). Figure $7 \mathrm{E}, \mathrm{F}$ depicts eABR recordings in $g p$ - $m r 02$ after 4 weeks of BDNF treatment (i.e., 6 weeks after deafening) and 2 weeks after cessation of BDNF treatment (i.e., 8 weeks after deafening). The $\mathrm{N}_{1}-\mathrm{P}_{2}$ amplitude did not decrease after cessation of BDNF treatment, and the threshold did not change.

\section{eABR amplitude}

Figure 8 shows the input-output curves of eABRs in normal-hearing animals, animals 6 weeks after deafening, animals after 4 weeks of BDNF treatment (i.e., 6 weeks after deafening), and animals 2 weeks after cessation of BDNF treatment. The amplitude of the $\mathrm{N}_{1}-\mathrm{P}_{2}$ complex was decreased in deafened animals for stimulus intensities of $252 \mu \mathrm{A}$ (unpaired $t$ test, $p<$ 0.05 ), $318 \mu \mathrm{A}$ (unpaired $t$ test, $p<0.01$ ), and $400 \mu \mathrm{A}$ (unpaired $t$ test, $p<0.001$ ) as compared to the amplitude in normal-hearing animals. At lower stimulus currents (100-200 $\mu \mathrm{A})$, the amplitude differences were smaller and not significant $(p>0.2)$. The

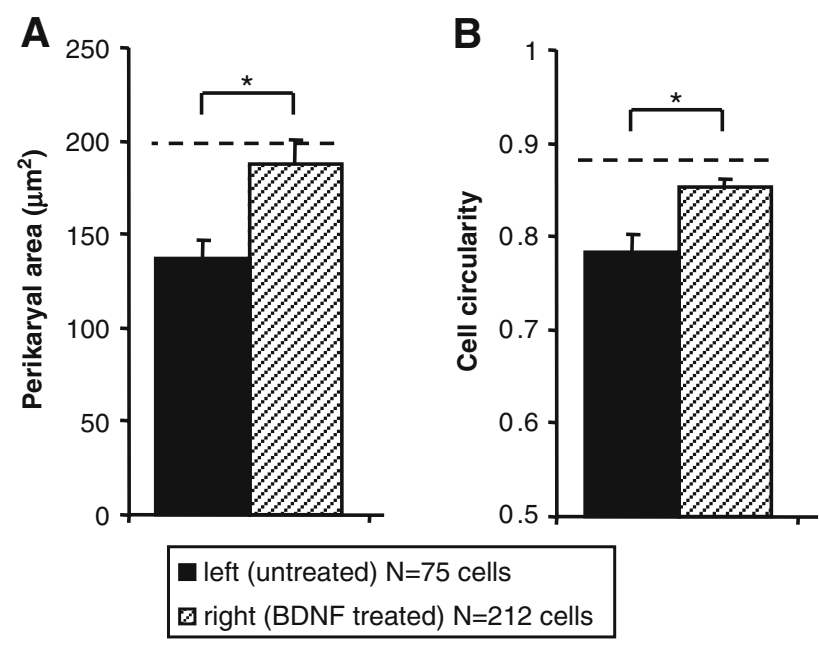

FIG. 6. Perikaryal area (A) and cell circularity (B) of SGCs in left untreated versus BDNF-treated cochleae. Only SGCs with an obvious nucleus in basal location B2 were included. Data were obtained by averaging all individual SGC measurements within one spiral ganglion, followed by averaging across cochleae. Dashed lines represent measurements in normal-hearing animals. $n$ the number of SGCs measured. Error bars: SEM. ${ }^{*} p<0.05$. 

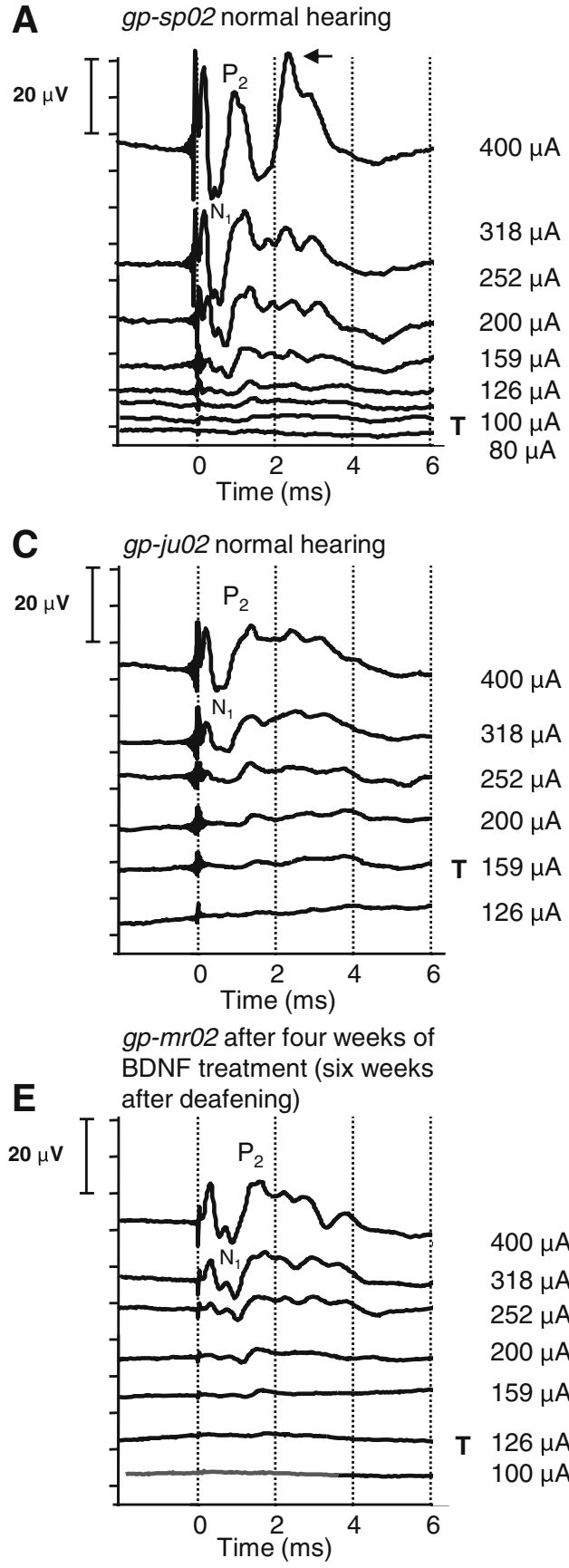

$400 \mu \mathrm{A}$

$318 \mu \mathrm{A}$

$252 \mu \mathrm{A}$

$200 \mu \mathrm{A}$

$159 \mu \mathrm{A}$

T $126 \mu \mathrm{A}$ $100 \mu \mathrm{A}$

FIG. 7. Recordings of representative eABRs evoked with current pulses of $400 \mu \mathrm{A}$ down to below threshold in two control animals (gp-sp02 and gp-ju02) and one experimental animal (gp-mrO2). Recordings of two control animals are depicted to illustrate the interanimal variability. A, C Recorded in normal-hearing condition, prior

amplitudes in BDNF-treated animals were near the amplitudes found in normal-hearing animals (unpaired $t$ test, $p>0.4$ ) and significantly larger than in the deafened animals for stimulus intensities of $318 \mu \mathrm{A}$ (unpaired $t$ test, $p<0.05$ ) and $400 \mu \mathrm{A}$ (unpaired $t$ test, $p<0.01)$. There was no significant change in amplitude at any current level after cessation of treatment (paired $t$ test, $p>0.1$ ).
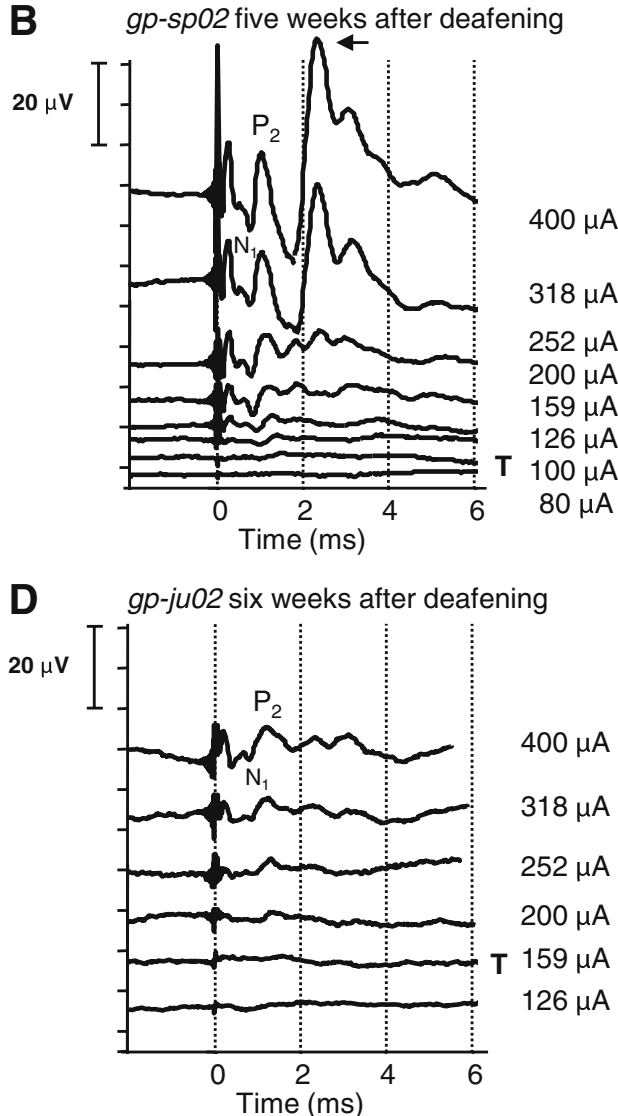

gp-mr02 two weeks after cessation of BDNF treatment

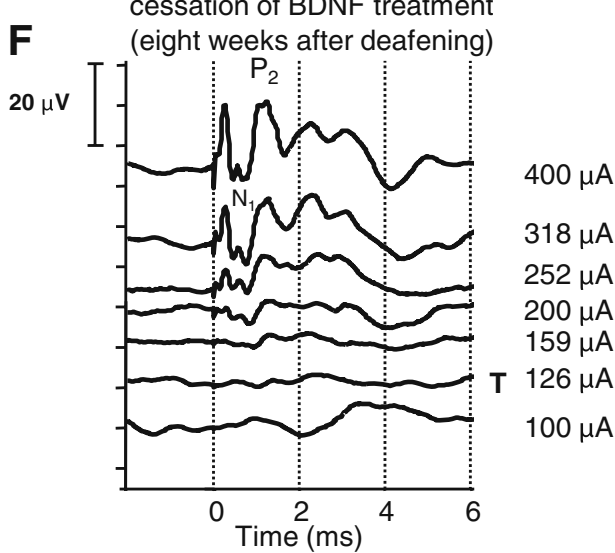

to deafening; B, D recorded 5 and 6 weeks after deafening, respectively. E Recorded after 4 weeks of BDNF treatment and $\mathbf{F}$ 2 weeks after cessation of the treatment. $T$ indicates the threshold of wave $N_{1}-P_{2}$. The arrow indicates the peak that probably reflects the digastric muscle response.

Figure 9 shows the mean time course of eABR amplitudes elicited with $400 \mu \mathrm{A}$ pulses for the BDNFtreated animals and the control animals. In both groups, amplitudes were increasing during the first 23 weeks after implantation. The control animals showed a gradual and significant decrease in amplitude after deafening. Six weeks after deafening, the amplitude was at $60 \%$ of the original value. The 


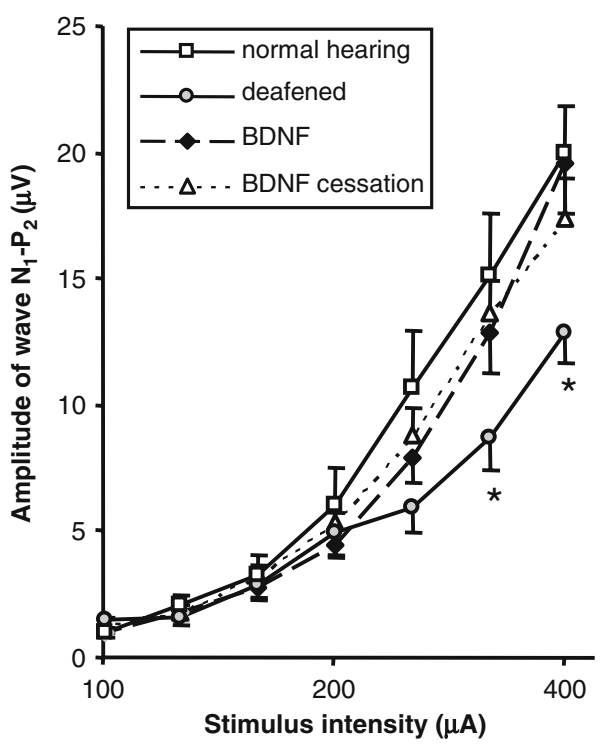

FIG. 8. Mean input-output functions of eABRs recorded in control animals before deafening (normal hearing, $n=12$ ) and 6 weeks after deafening $(n=9)$, and in the experimental animals immediately after 4 weeks of BDNF treatment $(n=5)$ and 2 weeks after cessation of BDNF treatment $(n=5)$. Error bars: SEM. ${ }^{*} p<0.05$ indicates statistical significant difference between the untreated animals 6 weeks after deafening and each of the other data points.

amplitude did not change significantly after cessation of BDNF treatment $(p>0.6)$. The eABR recordings in these animals also yielded threshold and latency data which will be described in the following sections.

\section{eABR threshold}

Figure 10 shows a moderate decrease in eABR threshold in the control group during the first weeks after implantation (rm ANOVA: $p<0.05$ ). The threshold in

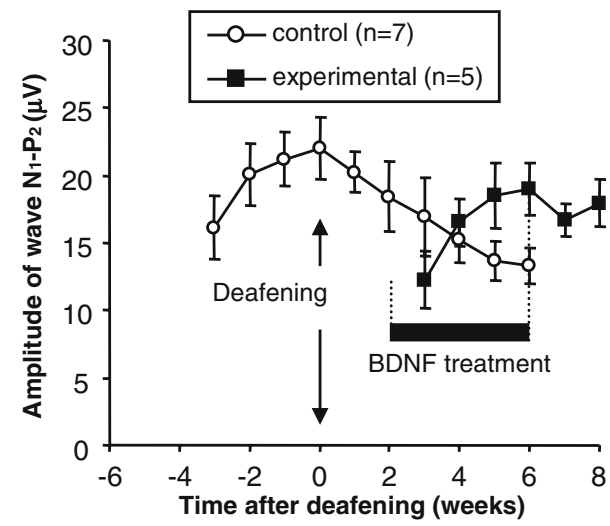

FIG. 9. Mean $\mathrm{N}_{1}-\mathrm{P}_{2}$ amplitudes of eABRs recorded to $400-\mu \mathrm{A}$ pulses in control $(n=7)$ and experimental $(n=5)$ animals. The control animals were implanted at -4 weeks and deafened at 0 weeks. The experimental animals were deafened at 0 weeks and implanted in the right cochlea at 2 weeks. Following implantation, the experimental animals received BDNF for a 4-week period (indicated with a horizontal bold bar). Error bars: SEM.

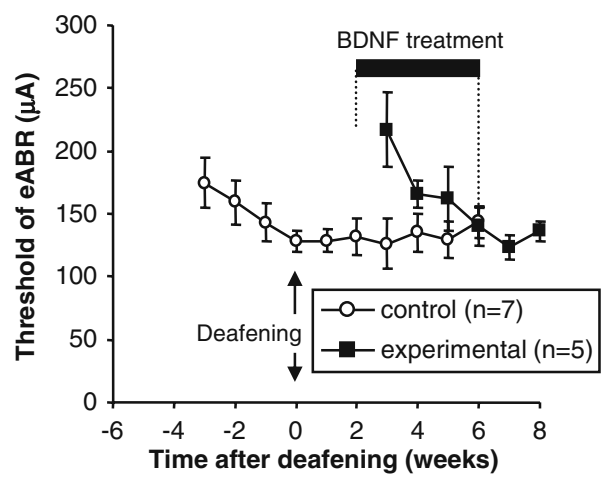

FIG. 10. Mean eABR thresholds observed for wave $N_{1}-P_{2}$ in control $(n=7)$ and experimental $(n=5)$ animals. The experimental animals were deafened at 0 weeks and implanted in the right cochlea at 2 weeks. Following implantation, the experimental animals received BDNF for a 4 -week period (indicated with a horizontal bold bar). Threshold criterion $2 \mu \mathrm{V}$; error bars: SEM.

these animals did not change significantly during 6 weeks after deafening ( $\mathrm{rm}$ ANOVA: $p>0.5$ ). The eABR threshold significantly decreased over time in the group temporarily treated with BDNF (rm ANOVA: $p<0.01$ ). After cessation of BDNF treatment, the eABR thresholds did not demonstrate any significant changes.

\section{eABR latency}

Figure 11 shows the time course of $\mathrm{N}_{1}$ latencies of eABRs to $400-\mu \mathrm{A}$ pulses for both the BDNF-treated and control animals. Deafening caused a prominent latency change, which occurred mostly in the first 2 weeks: first a decrease and then an increase. In the deafened condition, latencies were longer by about $0.15 \mathrm{~ms}$ than in the normal condition (paired $t$ test 6 weeks after deafening versus normal, $p<0.001$ ). In general, animals treated with BDNF demonstrated

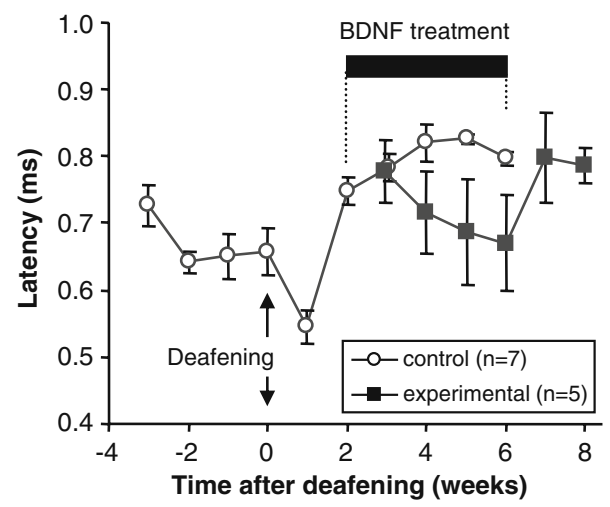

FIG. 11. Mean $\mathrm{N}_{1}$ latencies of eABRs evoked with 400- $\mu \mathrm{A}$ pulses, observed in control $(n=7)$ and experimental $(n=5)$ animals. The experimental animals were deafened at 0 weeks and implanted in the right cochlea at 2 weeks. Following implantation, the experimental animals received BDNF for a 4-week period (indicated with a horizontal bold bar). Statistical analyses within each group are not shown, but are described in the text. Error bars: SEM. 
latencies longer than normal. During the treatment period, the variance among animals was large, with some animals having longer latencies (as compared to the deafened controls) and others having shorter latencies (as compared to controls 1 week after deafening). After cessation of treatment, all animals had longer latencies, very similar to deafened controls and significantly longer than normal-hearing controls (unpaired $t$ test, $p<0.01$ ). Statistical analysis of the latencies performed on all animals (as in Fig. 8) showed similar outcomes.

\section{DISCUSSION}

The present study showed that temporary treatment with BDNF prevented degeneration of SGCs, which is commonly observed after inner hair cell loss in ototoxically deafened guinea pigs (Ylikoski et al. 1974; Webster and Webster 1981). Two weeks after cessation of BDNF treatment, SGC packing densities were as in cochleae of normal-hearing guinea pigs and three times greater than in the untreated contralateral cochleae (Fig. 5). Amplitudes of eABR wave $\mathrm{N}_{1}-\mathrm{P}_{2}$ in BDNF-treated animals were comparable to those in normal-hearing animals and larger than in untreated deafened animals.

\section{Preservation of SGCs after cessation of treatment}

In contrast to our findings, Gillespie et al. (2003) and Shepherd et al. (2008) reported that the number of SGCs was similar to that in untreated, contralateral cochleae, already 2 weeks after withdrawal of BDNF. In addition, Shepherd et al. (2008) reported that electrical stimulation after BDNF treatment only partially prevented degeneration of SGCs in the basal turn. The differences between their studies and our study that may contribute to the contrasting results are: (1) recordings of the eABRs, (2) concentration of BDNF, (3) method of cessation of BDNF treatment, and (4) period of deafness before the start of the neurotrophic treatment.

1. We recorded eABRs, while Gillespie et al. (2003) did not. The brief $(\sim 20 \mathrm{~min})$ stimulation at $10 \mathrm{pulses} / \mathrm{s}$ twice per week might have provided some neurotrophic support enhancing the survival of SGCs after cessation of BDNF treatment. Prevention of SGC degeneration in deafened animals, solely due to eABR recordings, has been previously reported (Miller and Altschuler 1995; Mitchell et al. 1997). Chikar et al. (2008) found enhanced SGC survival 12 weeks after a single inoculation with an adenovirus suspension encoding for BDNF (Ad.BDNF), and they argued that eABR recordings contributed to this. Maruyama et al. (2008) demonstrated enhanced survival of SGCs after cessation of intracochlear infusion of GDNF. Similar to our study, brief electrical stimulation during eABR recordings was provided once a week. A synergistic effect of BDNF treatment in combination with chronic electrical stimulation has also been reported (Kanzaki et al. 2002; Shepherd et al. 2005; Song et al. 2009), indicating that synergy of eABR recordings and BDNF treatment might be present. Finally, we found that eABRs prevented decrease of SGC size, which might indicate some trophic effect (Richardson et al. 2005). Based on the data described above, one would argue that eABR recordings explain the discrepancy between our study and their studies. However, the following arguments point against this effect of eABRs. First, our results demonstrated that eABR recordings alone did not affect the SGC packing density. Second, Shepherd et al. (2008) reported that electrical stimulation only prevented SGC degeneration in the basal cochlear turn, whereas we found persistent survival in all cochlear locations examined (2.5 cochlear turns).

2. In our study, a BDNF concentration of $100 \mu \mathrm{g} / \mathrm{ml}$ was chosen, and in their studies $62.5 \mu \mathrm{g} / \mathrm{ml}$. Because concentrations in the range of $50-100 \mu \mathrm{g} / \mathrm{ml}$ are equally effective, as judged immediately after treatment (Gillespie et al. 2004; Yamagata et al. 2004; Wise et al. 2005; Miller et al. 2007; Agterberg et al. 2008), and even lower concentrations have been reported to be effective $(50 \mathrm{ng} / \mathrm{ml}$; Miller et al. 1997), it is unlikely that differences in concentration may explain the conflicting results.

3. We cut off the cannula to stop the BDNF flow exactly 4 weeks after the start of the treatment. It cannot be excluded that residual BDNF in the cannula $(\sim 10 \mu \mathrm{l})$ could diffuse in the cochlea resulting in a lasting effect on SGC survival. This seems to be unlikely since the tip of the cannula $(0.12 \mathrm{~mm})$ was very small, and we observed that the tip was obstructed when we killed the animals for histology (2 weeks after the flow rate stopped). In their studies, the mini-osmotic pumps were not removed after cessation of BDNF treatment. Delivery of BDNF stopped 4 weeks after implantation because the lifetime of the pump was limited to 4 weeks. However, an empty reservoir of the osmotic pump will result in an increased osmotic pressure, which might eventually cause damage to pump and cannula. The effect on the cochlea is hard to assess, but one cannot exclude for instance withdrawal or contamination of perilymph jeopardizing the condition of the SGCs.

4. In their studies, BDNF treatment started 5 days after deafening, which is before loss of SGCs, whereas in 
our study, it started after 2 weeks when SGC degeneration has started (Versnel et al. 2007).

Considering the four issues addressed above, we propose $\mathrm{ABR}$ recordings as the most likely candidate to explain the discrepancy between our data and those of Gillespie et al. (2003). However, we cannot exclude any of the other three arguments.

Although the lasting effect of the neurotrophic treatment is evident in the present study (Fig. 5), this effect is only evaluated 2 weeks after cessation. Degeneration after deafening is not pronounced after 2 weeks ( $\sim 80 \%$ of normal density, Versnel et al. 2007$)$. Also the decline in eABR amplitude after deafening is rather slow (Fig. 9). Therefore, to investigate, both histologically and functionally, whether degeneration after cessation of BDNF treatment is slower than degeneration after deafening without intervention, it is important to apply longer periods of survival.

\section{Morphology of SGCs after cessation of BDNF treatment}

Immediately after BDNF treatment, SGCs are larger than SGCs in normal-hearing animals (Shepherd et al. 2005; Richardson et al. 2005; Agterberg et al. 2008). We found that 2 weeks after cessation SGCs had a normal size (while larger than in the untreated contralateral cochlea). This implies that the perikaryal area decreased after cessation from larger-than-normal, to normal values. The question remains if the back-to-normal area is an indication of a normal condition. On the other hand, the decrease of cell size following cessation may be an early indication for initiation of a degenerative process (Staecker et al. 1996; Leake et al. 1999; Agterberg et al. 2008). To answer this last question, the survival of SGCs needs to be investigated for longer periods after cessation of the neurotrophic treatment.

\section{eABR amplitudes}

We applied suprathreshold amplitudes as a measure of excitability of the auditory nerve, since the amplitude reflects the summed neural firings of a large population of SGCs, whereas the more commonly used parameter threshold reflects the function of only the most sensitive auditory nerve fibers. Indeed, at least in case of monopolar stimulation, the amplitude of early eABR waves has been found to correlate well with the number of SGCs (Smith and Simmons 1983; Hall 1990) whereas correlations between eABR threshold and nerve survival were weak (Smith and Simmons 1983). Note that for bipolar stimulation a better correlation was found for threshold measures than for amplitude measures (Miller et al. 1994).
The normal eABR amplitude in BDNF-treated animals indicates that treated SGCs responded as normal to electric stimuli. It should be noted that the amplitude after deafening was smaller, probably because less SGCs were present, thus implicating that the remaining untreated cells responded normally. Our data agree with the results of Maruyama et al. (2008) who reported that amplitudes in neurotrophically treated animals were larger than in untreated animals and were stable after cessation of the treatment.

A decrease of eABR threshold was found the first weeks after implantation in the normal-hearing condition. This might be due to mechanisms as tissue growth around the electrodes and recovery from insertion-induced trauma (Su et al. 2008). We assume the threshold decrease found in the animals treated with BDNF was rather due to these mechanisms than to the preservation of SGCs mediated by the BDNF.

Our threshold data do not agree with other investigators who reported lower thresholds (by 4-9 dB) in deafened guinea pigs treated with neurotrophins as compared to deafened and untreated guinea pigs (Shinohara et al. 2002; Yamagata et al. 2004; Shepherd et al. 2005; Chikar et al. 2008; Maruyama et al. 2008). In these studies, the decrease in eABR threshold corresponded with enhanced survival of SGCs after neurotrophic treatment. Contrary to these studies, in which eABR thresholds were often assessed on the basis of visual inspection of peak 3 and recordings were performed in anesthetized animals, we assessed the eABR threshold on the basis of interpolation of $\mathrm{N}_{1}-\mathrm{P}_{2}$ amplitudes and we recorded in awake animals. Possibly, the difference in peaks analyzed explains the different threshold data as Mitchell et al. (1997) reported that the threshold of the late eABR peaks in deafened animals decreased over time, while early peak thresholds did not significantly change. Further, the state of the animal plays a role in that the threshold increases with anesthesia as was found for aABRs in mice by Van Looij et al. (2004). The effects of anesthesia may be nonlinear and differ between various experimental conditions.

\section{eABR latencies}

The eABR $\mathrm{N}_{1}$ latencies were longer in deafened and BDNF-treated animals than in normal-hearing animals (Fig. 11). The initial latency decrease after deafening may be caused by degeneration of dendrites and a loss of spontaneous activity, and the subsequent increase can be attributed to a reduction of the number of myelin layers, which follows dendritic degeneration (Leake and Hradek 1988). The BDNF-treated nerve cells have a similar degree of myelination as untreated cells (Agterberg et al. 2008; Glueckert et al. 2008), and this might explain the similar latencies of BDNF-treated and untreated animals. 


\section{Clinical implications}

Our results demonstrate that SGC survival in deafened animals, in both morphological and functional sense, is not reduced after cessation of intracochlear application of BDNF. The enhanced survival of SGCs is a promising finding for cochlear implant candidates since there are currently no potential methods available to provide neurotrophins for periods that extend the order of months. Lifelong neurotrophic treatment via an osmotic pump is not clinically preferable because of repetitive invasive manipulations needed to replace the pump and high infection risks.

Before neurotrophic treatment can be considered a clinical option, it is important to investigate in animal studies whether neurotrophic treatment does actually improve the sound perception performance with a cochlear implant. A major topic that needs to be further explored is the assumption that more and better functioning SGCs would result in a better performance of cochlear implant users. Alternative to this hypothesis, it might be that a minimum number of SGCs is required for electrical hearing, and that an increase above this minimum would have little functional impact (Blamey 1997). Clinical data do not support the assumption that more SGCs would lead to a better behavioral performance (Nadol et al. 2001; Khan et al. 2005; Fayad and Linthicum 2006; Nadol and Eddington 2006). These data indicate that abnormalities in the central auditory pathways are at least as important as SGC survival in limiting the performance of cochlear implant users (Nadol et al. 2001). Furthermore, the overall performance of cochlear implant users improves markedly within weeks as a result of implant use, confirming the role of the central auditory system and its plasticity (Kral and Eggermont 2007). Still, we, and others, think that it is unlikely that SGC loss does not affect the performance with a cochlear implant and that the human data sets are still too limited to conclude that the extent of SGC survival does not influence cochlear implant performance (Khan et al. 2005; Leake et al. 2008). Moreover, it should be considered that with improved cochlear implant technology the number of SGCs might become more important (Green et al. 2008).

\section{ACKNOWLEDGMENTS}

The authors would like to thank Leone Nijman and Rik Mansvelt-Beck for their excellent practical and technical assistance, Ferry Hendriksen for assisting with histology, and René van de Vosse for developing the recording and analysis software. We thank Victor Wiegant for his suggestions on the manuscript. This study was supported by the HeinsiusHoubolt Foundation.
Open Access

This article is distributed under the terms of the Creative Commons Attribution Noncommercial License which permits any noncommercial use, distribution, and reproduction in any medium, provided the original author(s) and source are credited.

\section{REFERENCES}

Agterberg MJH, Versnel H, de Groot JCMJ, Smoorenburg GF, AlBERs FWJ, KuIS SFL. Morphological changes in spiral ganglion cells after intracochlear application of brain-derived neurotrophic factor in deafened guinea pigs. Hear. Res. 244:25-34, 2008.

BLAMEY P. Are spiral ganglion cell numbers important for speech perception with a cochlear implant? Am. J. Otol. 18(suppl):1112, 1997.

Brown JN, Miller JM, Altschuler RA, Nuttall AL. Osmotic pump implant for chronic infusion of drugs into the inner ear. Hear. Res. 70:167-172, 1993.

Chikar Ja, Colesa DJ, Swiderski DL, Polo AD, Raphael Y, Pfingst BE. Over-expression of BDNF by adenovirus with concurrent electrical stimulation improves cochlear implant thresholds and survival of auditory neurons. Hear. Res. 245:24-34, 2008.

De Groot JCMJ, Veldman JE, Huzing EH. An improved fixation method for guinea pig cochlear tissues. Acta Otolaryngol 104:234-242, 1987.

FAYAD JN, LinTHICUM FH, JR. Multichannel cochlear implants: relation of histopathology to performance. Laryngoscope 116:13101320, 2006.

Gillespie LN, ShePherd RK. Clinical application of neurotrophic factors: the potential for primary auditory neuron protection. Eur. J. Neurosci. 22:2123-2133, 2005.

Gillespie LN, Clark GM, Bartlett PF, Marzella PL. BDNF-induced survival of auditory neurons in vivo: cessation of treatment leads to accelerated loss of survival effects. J. Neurosci. Res. 71:785790, 2003.

Gillespie LN, Clark GM, Marzella PL. Delayed neurotrophin treatment supports auditory neuron survival in deaf guinea pigs. Neuroreport 15:1121-1125, 2004.

Glueckert R, Bitsche M, Miller JM, Zhu Y, Prieskorn DM, Altschuler RA, Schrott-Fischer A. Deafferentation-associated changes in afferent and efferent processes in the guinea pig cochlea and afferent regeneration with chronic intrascalar brain-derived neurotrophic factor and acidic fibroblast growth factor. J. Comp. Neurol. 507:1602-1621, 2008.

Green SH, Altschuler RA, Miller JM. Cell death and cochlear protection. In: Schacht J, Popper AN, Fay RR (eds) Auditory Trauma, Protection, and Repair. New York, Springer, pp. 275319, 2008.

HALL RD. Estimation of surviving spiral ganglion cells in the deaf rat using the electrically evoked auditory brainstem response. Hear. Res. 45:123-136, 1990.

Hartshorn DO, Miller JM, Altschuler RA. Protective effect of electrical stimulation in the deafened guinea pig cochlea. Otolaryngol. Head Neck Surg. 104:311-319, 1991.

Kanzaki S, Stover T, Kawamoto K, Prieskorn DM, Altschuler RA, MiLLER JM, RAPHAEL Y. Glial cell line-derived neurotrophic factor and chronic electrical stimulation prevent VIII cranial nerve degeneration following denervation. J. Comp. Neurol. 454:350360, 2002.

Khan AM, Handzel O, Burgess BJ, Damian D, Eddington DK, Nadol JB. Is word recognition correlated with the number of surviving spiral 
ganglion cells and electrode insertion depth in human subjects with cochlear implants? Laryngoscope 115:672-677, 2005.

Kral A, Eggermont JJ. What's to lose and what's to learn: development under auditory deprivation, cochlear implants and limits of cortical plasticity. Brain Res. 56:259-269, 2007.

Leake PA, Hradek GT. Cochlear pathology of long term neomycin induced deafness in cats. Hear. Res. 33:11-33, 1988.

Leake PA, Hradek GT, Rebscher SJ, SNyder RL. Chronic intracochlear electrical stimulation induces selective survival of spiral ganglion neurons in neonatally deafened cats. Hear. Res. 54:251-271, 1991.

Leake PA, Hradek GT, SNyder RL. Chronic electrical stimulation by a cochlear implant promotes survival of spiral ganglion neurons after neonatal deafness. J. Comp. Neurol. 412:543-562, 1999.

Leake PA, Stakhovskaya O, Hradek GT, Hetherington AM. Factors influencing neurotrophic effects of electrical stimulation in the deafened developing auditory system. Hear. Res. 242:86-99, 2008.

LOUSTEAU RJ. Increased spiral ganglion cell survival in electrically stimulated, deafened guinea pig cochleae. Laryngoscope 97:836-842, 1987.

Mansour-Robaey S, Clarke DB, Wang yC, Bray GM, Aguayo AJ. Effects of ocular injury and administration of brain-derived neurotrophic factor on survival and regrowth of axotomized retinal ganglion cells. Proc. Natl. Acad. Sci. U. S. A. 91:16321636, 1994.

Maruyama J, Miller JM, Ulfendahl M. Glial cell line-derived neurotrophic factor and antioxidants preserve the electrical responsiveness of the spiral ganglion neurons after experimentally induced deafness. Neurobiol. Dis. 29:14-21, 2008.

Miller JM, Altschuler RA. Effectiveness of different electrical stimulation conditions in preservation of spiral ganglion cells following deafness. Ann. Otol. Rhinol. Laryngol. Suppl. 166:5760, 1995.

Miller CA, Abbas PJ, Robinson BK. The use of long-duration current pulses to assess nerve survival. Hear. Res. 78:11-26, 1994.

Miller JM, Chi DH, O’Keeffe LJ, Kruszka P, Raphael Y, Altschuler RA. Neurotrophins can enhance spiral ganglion cell survival after inner hair cell loss. Int. J. Dev. Neurosci. 15:631-643, 1997.

Miller JM, Le Prell CG, Prieskorn DM, Wys NL, Altschuler RA. Delayed neurotrophin treatment following deafness rescues spiral ganglion cells from death and promotes regrowth of auditory nerve peripheral processes: effects of brain-derived neurotrophic factor and fibroblast growth factor. J. Neurosci. Res. 85:1959-1969, 2007.

Mitchell A, Miller JM, Finger PA, Heller JW, Raphael Y, Altschuler RA. Effects of chronic high-rate electrical stimulation on the cochlea and eighth nerve in the deafened guinea pig. Hear. Res. 105:30-43, 1997.

Montero CN, Hefti F. Rescue of lesioned septal cholinergic neurons by nerve growth factor: specificity and requirement for chronic treatment. J. Neurosci. 8:2986-2999, 1988.

Nadol JB, JR, Eddington DK. Histopathology of the inner ear relevant to cochlear implantation. Adv. Otorhinolaryngol 64:3149, 2006.

Nadol JB, Jr, Shiao JY, Burgess BJ, Ketten DR, Eddington DK, Gantz BJ, Kos I, Montandon P, Coker NJ, Roland JTJ, Shallop JK. Histopathology of cochlear implants in humans. Ann. Otol. Rhinol. Laryngol. 110:883-891, 2001.

Pettingill LN, Richardson RT, Wise AK, O'Leary SJ, Shepherd RK. Neurotrophic factors and neural prostheses: potential clinical applications based upon findings in the auditory system. IEEE Trans. Biomed. Eng. 54:1138-1148, 2007.

Richardson RT, O'leary S, Wise A, Hardman J, Clark G. A single dose of neurotrophin-3 to the cochlea surrounds spiral ganglion neurons and provides trophic support. Hear. Res. 204:37-47, 2005.

Romand R, Romand MR. The spiral ganglion. In: Friedmann I, Ballantyne J (eds) Ultrastructural Atlas of the Inner Ear. London, Butterworths, pp. 165-183, 1984.

SHEPHERD RK, XU J. A multichannel scala tympani electrode array incorporating a drug delivery system for chronic intracochlear infusion. Hear. Res. 172:92-98, 2002.

Shepherd RK, Coco A, Epp SB, Crook JM. Chronic depolarization enhances the trophic effects of brain-derived neurotrophic factor in rescuing auditory neurons following a sensorineural hearing loss. J. Comp. Neurol. 486:145-158, 2005.

Shepherd RK, Coco A, Epp SB. Neurotrophins and electrical stimulation for protection and repair of spiral ganglion neurons following sensorineural hearing loss. Hear. Res. 242:100-109, 2008.

Shinohara T, Bredberg G, Ulfendahl M, Pyykko I, Olivius NP, Kaksonen R, Lindstrom B, Altschuler R, Miller JM. Neurotrophic factor intervention restores auditory function in deafened animals. Proc. Natl. Acad. Sci. U. S. A. 99:1657-1660, 2002.

Smith L, Simmons FB. Estimating eighth nerve survival by electrical stimulation. Ann. Otol. Rhinol. Laryngol. 92:19-23, 1983.

Song BN, Li YX, Han DM. Delayed electrical stimulation and BDNF application following induced deafness in rats. Acta Otolaryngol 129:142-154, 2009.

Spoendin H. Retrograde degeneration of the cochlear nerve. Acta Otolaryngol 79:266-275, 1975

Staecker H, Kopke R, Malgrange B, Lefebvre P, Van De Water TR. NT-3 and/or BDNF therapy prevents loss of auditory neurons following loss of hair cells. Neuroreport 7:889-894, 1996.

Su GL, Colesa DJ, Pfingst BE. Effects of deafening and cochlear implantation procedures on postimplantation psychophysical electrical detection thresholds. Hear. Res. 241:64-72, 2008.

Van Looij MAJ, Liem SS, Van der Burg H, Van der Wees J, De Zeeuw CI, VAN ZANTEN BGA. Impact of conventional anesthesia on auditory brainstem responses in mice. Hear. Res. 193:75-82, 2004.

Versnel H, Agterberg MJH, de Groot JCMJ, Smoorenburg GF, Klis SFL. Time course of cochlear electrophysiology and morphology after combined administration of kanamycin and furosemide. Hear. Res. 231:1-12, 2007.

Webster M, Webster DB. Spiral ganglion neuron loss following organ of corti loss: a quantitative study. Brain Res. 212:17-30, 1981.

West BA, BRummett RE, Himes DL. Interaction of kanamycin and ethacrynic acid. Severe cochlear damage in guinea pigs. Arch. Otolaryngol 98:32-37, 1973.

Wise AK, Richardson R, Hardman J, Clark G, O'Leary S. Resprouting and survival of guinea pig cochlear neurons in response to the administration of the neurotrophins brain-derived neurotrophic factor and neurotrophin-3. J. Comp. Neurol. 487:147-165, 2005.

Yamagata T, Miller JM, Ulfendahl M, Olivius NP, Altschuler RA, Pyykko I, Bredberg G. Delayed neurotrophic treatment preserves nerve survival and electrophysiological responsiveness in neomycin-deafened guinea pigs. J. Neurosci. Res. 78:75-86, 2004.

Ylikoski J, Wersall J, BJorkroth B. Degeneration of neural elements in the cochlea of the guinea-pig after damage to the organ of corti by ototoxic antibiotics. Acta Otolaryngol. Suppl. 326:23-41, 1974. 